\title{
Frank Pfirsching
}

\section{Portfoliomanagement für selbstgenutzte Immobilien - die vernachlässigte Controllingfunktion}

Asset Management; Bedarfsplanung; Controlling; Immobilien; Portfoliomanagement; Public Real Estate Management (PREM)

Portfoliomanagement für selbstgenutzte Immobilien ist eine Controllingfunktion im Sinne der modernen Controllingdefinition als Koordinations- und Rationalitätssicherungsfunktion für die Unternehmensführung. Bei öffentlichen Unternehmen und Haushalten wird Portfoliomanagement häufig noch vernachlässigt. Der vorliegende Kurzbeitrag greift dieses Defizit auf und gibt einen Überblick über den Portfoliomanagementansatz, den zugrunde liegenden Managementprozess und zwischenzeitlich entwickelte Instrumente zum effektiven Management von selbstgenutzten Immobilien.

\section{Problemstellung}

Controlling als Koordinationsfunktion in der Unternehmensführung hat seit den ersten Veröffentlichungen Anthonys in den 1960er Jahren große Bedeutung für die Steuerung von Unternehmen (Schäffer 2004, S. 4; synonyme Verwendung zu ,management control“ im Englischen). In den 1970er Jahren übertrug Anthony seine Ansätze auf gemeinnützige Organisationen und spätestens mit den Ansätzen zur Neuen Steuerung zog der Controllinggedanke auch in deutsche öffentliche Unternehmen und Haushalte ein.

In einer parallelen Entwicklung übertrug Duckworth (1993) das Controllingkonzept auf selbstgenutzte Immobilien, d. h. Immobilien von Eigentümern, die diese Liegenschaften nur besitzen, um sie selbst nutzen zu können. Er setzte ,real property portfolio management“" gleich mit Anthonys ,management control“ (Duckworth 1993, S. 497).

Die öffentliche Hand unterschätzt dabei die Bedeutung ihrer selbstgenutzten Immobilien (wie z. B. Verwaltungsgebäude) und der daraus resultierenden Kosten und Risiken. Ecke schätzt den Anteil der Immobilienkosten auf 15 bis $20 \%$ des Verwaltungshaushalts (Ecke 2004, S. 2).

Trotz der theoretischen Vorarbeiten hängt die Einführung des Controllings als Führungsinstrument für selbstgenutzte Immobilien, das Portfoliomanagement, weit hinter der Verbreitung von allgemeinen Controllingansätzen zurück. Wahrscheinlicher Grund dafür ist, dass die Komplexität des Managements größerer Immobilienbestände von den Entscheidern unterschätzt wird, da die Verwaltung von Einzelimmobilien sehr effektiv durch den Nutzer selbst erledigt werden kann. Wird aber eine kritische Masse an Liegenschaften erreicht, so wird ohne eine zentrale Gesamtsteuerung und ein Controlling auf Portfolioebene die Maximierung des funktionalen und finanziellen Wertes der Immobilien ver- 
fehlt (Varcoe 2000, S. 113). Dies trifft heute noch auf Unternehmen und vor allem auf öffentliche Unternehmen und Haushalte zu.

In den Jahren 1998 und 1999 bildete sich in den USA die Corporate Real Estate Portfolio Alliance, eine Initiative von Forschern, Unternehmen und der General Services Administration (GSA) der US-amerikanischen Bundesverwaltung, mit dem Anspruch, einen Managementansatz zu entwickeln, der die folgenden drei Betrachtungsperspektiven von selbstgenutzten Immobilien verbindet und den Mehrwert der Immobilien maximiert (Varcoe 2000, S. 113 f.):

- Immobilie als Vermögensgegenstand (Unternehmensfinanzierungsperspektive).

- Immobilie aus Immobilienmarktsicht.

- Immobilie als Produktionsfaktor.

Die Alliance, Forscher und Praktiker wollen dabei den folgenden Defiziten begegnen:

- Keine gemeinsame Betrachtung der drei oben genannten Perspektiven, sondern Fokussierung auf Einzelobjektebene, dadurch Vernachlässigung der Entwicklung einer Portfoliostrategie in Abhängigkeit von der Unternehmensstrategie (Roulac 2001, S. 130).

- Ineffektiver Bottom-Up-Ansatz: Individuelle Nutzerwünsche und das Vorhandensein des Einzelobjekts bestimmen die Strategie, nicht die Strategie die Objektauswahl (Top-Down-/ Portfolioansatz) (Van der Schaaf/De Puy 2001, S. 152).

- Fehlende Entscheidungs- und Umsetzungsinstrumente zum Umgang mit folgenden Herausforderungen: ${ }^{1}$

- Konflikten zwischen unterschiedlichen Interessensgruppen, ${ }^{2}$

- Fehlende Fähigkeit der Nutzer zur Langzeitplanung ihrer Bedarfe (Van der Schaaf/De Puy 2001, S. 157),

- Notwendigkeit der Antizipation zukünftiger Entwicklungen aufgrund des Konflikts zwischen sich ständig ändernden Umweltbedingungen (Marktbedingungen) und den Eigenschaften der Immobilie als statischem Vermögensgegenstand (lange Bauzeit, lange Amortisationsdauer) (Van der Schaaf/De Puy 2001, S. 152),

- Getroffene Strategieentscheidungen geben keine expliziten, klaren Handlungsanweisungen für spätere Entscheidungen und Umsetzung vor (Van der Schaaf/De Puy 2001, S. 153).

Während in Deutschland zumindest privatwirtschaftliche Unternehmen diese Ansätze einführten, werden sie bei öffentlichen Unternehmen und Haushalten noch vernachlässigt (Schulte et al. 2006, S. 29).

Dagegen nahmen GSA und weitere internationale Immobilienverwalter die Vorschläge der Alliance in einem gemeinsamen Forschungsprojekt mit der TU Delft auf und entwi-

1 Evers/Van der Schaaf/Dewulf 2002, S. 79: Folge: zu kurzer Planungshorizont, keine kontinuierliche Anpassung an Nutzer- und Marktbedarfe.

2 Eigentümerfunktion, Nutzerfunktion und Verwalterfunktion selbst (Van der Schaaf/De Puy 2001, S. 151; The National Academy of Sciences 2004, S. 44; Pfirsching 2007, S. 9). 
ckelten auf die öffentliche Verwaltung zugeschnittene Instrumente (Evers/Van der Schaaf/Dewulf 2002, S. 79).

Bei den genannten Defiziten werden sich auch deutsche öffentliche Unternehmen und Haushalte wiederfinden (Schulte et al. 2006, S. 29). In vielen Fällen gibt es sogar keine Portfoliomanagement- bzw. Controllingfunktion für die selbstgenutzten Immobilien. ${ }^{3}$ Weiterhin haben gerade der Bund, Bundesländer und Großstädte die größten und komplexesten Immobilienbestände mit häufig mehreren Tausend Objekten. Somit sollte der Handlungsbedarf gerade hier besonders groß sein.

In Form eines kurzen Überblicks werden nun der Portfoliomanagementansatz der Alliance und zwischenzeitlich entwickelte Instrumente vorgestellt, um die Umsetzung von Portfoliomanagement als Controllingfunktion bei öffentlichen Unternehmen und Haushalten anzuregen.

\section{Definition}

Ein erstes Ergebnis der Zusammenarbeit der Corporate Real Estate Portfolio Alliance war die Festlegung einer Definition für Portfoliomanagement von selbstgenutzten Immobilien:

"Managing real properties as a group in order to achieve greater corporate benefits from them as productive working environment assets, financial assets and strategic assets, above the benefits derived from managing them individually" (Varcoe 1999, S. 116).

Diese Sichtweise sollte für alle Organisationen gelten, die als „enterprises“ bezeichnet werden. Diese Bezeichnung ist synonym zu verwenden mit dem deutschen Begriff „Selbstnutzer“ (Pfirsching 2007, S. 9). Die Immobilieneigentümergruppe der „enterprises" beinhaltet private Unternehmen, öffentliche Unternehmen und öffentliche Verwaltungen und Agenturen (Szigeti/Davis 2000, S. 1).

In einem zweiten Schritt nahm die Alliance eine Einordnung der Funktion Portfoliomanagement in die Organisation des Selbstnutzers vor (siehe Abbildung 1).

3 Typische Symptome dafür sind: Keine zentrale Transparenz über Objektanzahlen, Flächen, Leerstände, Werte, zeitliche Nutzungsbedarfe. 


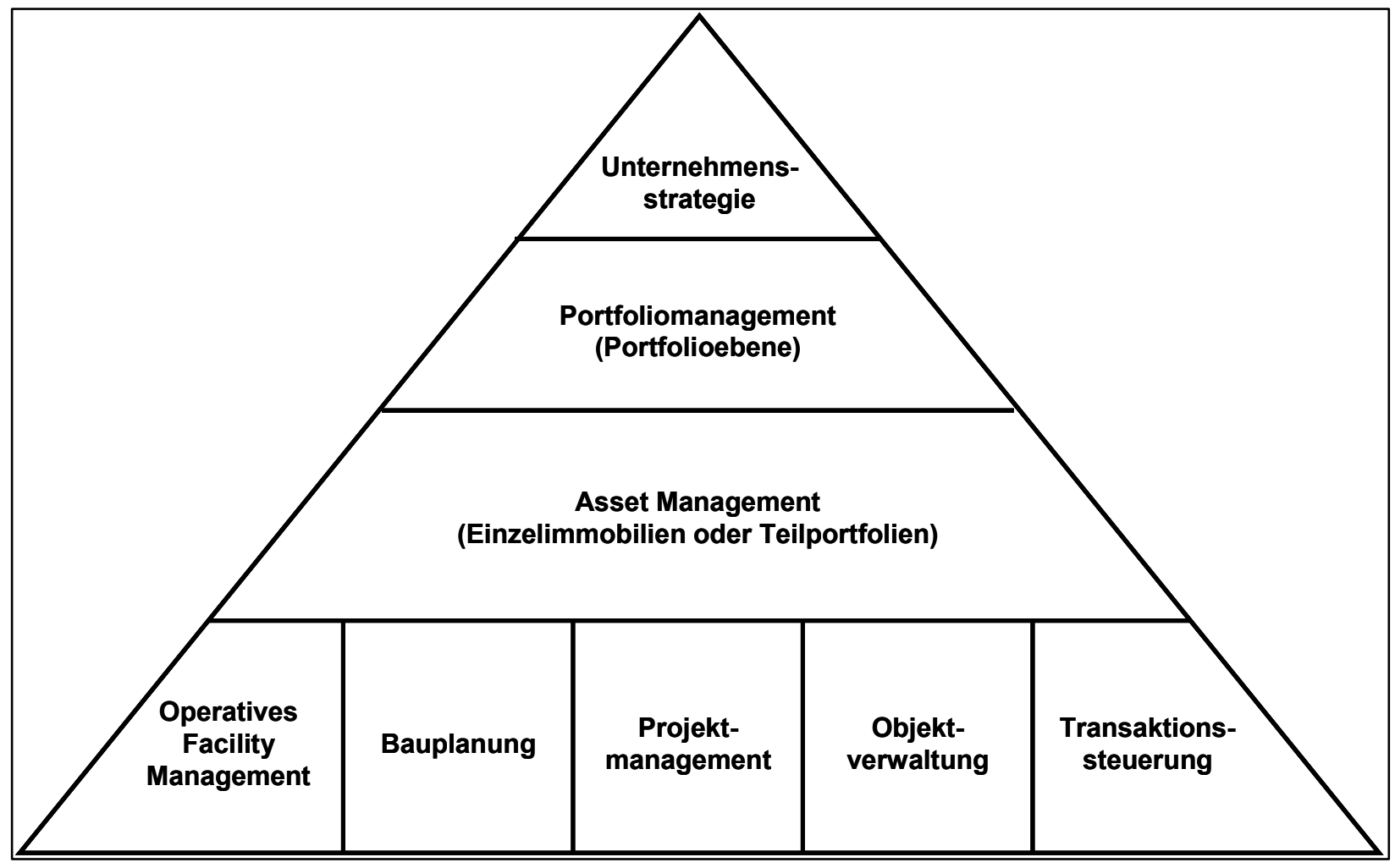

Abb. 1: Organisatorische Einordnung von Portfoliomanagement, Asset Management und untergeordneter operativer Funktionen

Quelle: $\quad$ Szigeti/Davis 2000, S. 2

In diesem Sinne übernimmt das Portfoliomanagement die Rolle des immobilienwirtschaftlichen Key Account-Betreuers der unternehmensweiten Strategieeinheit sowie der Nutzer und stellt die für die gesamte Organisation notwendigen Immobilienflächen und -funktionen zur Verfügung. Es beschäftigt sich dabei mit dem ganzen Portfolio. In der Steuerung ist die wesentliche Aufgabe die Vorgabe finanzieller Ziele gegenüber dem Asset Management. Das Asset Management steuert dann die operative Bewirtschaftung und beschäftigt sich dabei mit Einzelobjekten oder Teilportfolien (z. B. alle Liegenschaften in einer Stadt). Das Asset Management ,,beauftragt“" so die Maßnahmen im Sinne der Zielvorgaben des Portfoliomanagements, die anschließend durch die operativen Einheiten umgesetzt werden (Szigeti/Davis 2000, S. 4 ff.).

\section{Prozess und Funktionen}

Ein weiteres Ergebnis der Alliance-Arbeit ist die Erhebung und Dokumentation des Portfoliomanagementprozesses. Dabei werden fünf Phasen unterschieden (siehe Abbildung 2). 


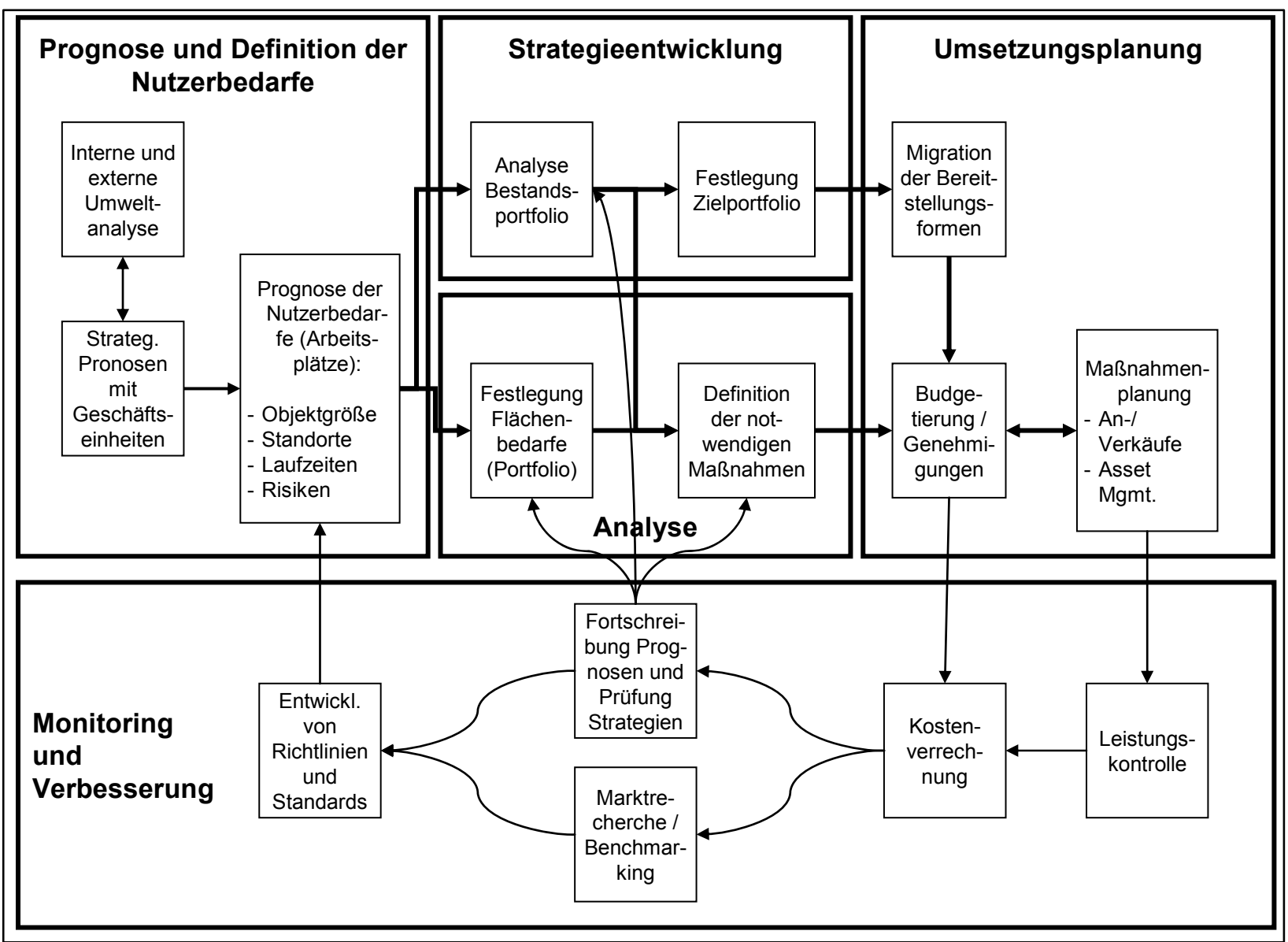

Abb. 2: Der Portfoliomanagementprozess für selbstgenutzte Immobilien

Quelle: $\quad$ Varcoe 2000, S. 120

In der ersten Phase (Prognose und Definition der Nutzerbedarfe) werden auf Basis einer Umweltanalyse durch den Portfoliomanager und strategischer Prognosen mit den Kerngeschäftseinheiten konkrete Prognosen für die Immobilienflächenbedarfe getroffen.

Dann erfolgt in der Analysephase der Abgleich mit den tatsächlich vorhandenen Immobilienressourcen, um in der Strategieentwicklungsphase Anpassungsbedarfe des Portfolios definieren zu können.

Diese Maßnahmen werden danach in der Phase der Umsetzungsplanung detailliert geplant, budgetiert und in ein Maßnahmenprogramm für die operativen Einheiten überführt. In der kontinuierlich parallel verlaufenden Monitoring- und Verbesserungsphase werden Richtlinien und Standards als Vorgabe für die Arbeit der Asset und Facility Manager definiert, die eigenen Prognosen und Strategien vor dem Hintergrund sich ständig ändernder Umweltbedingungen überprüft und Leistungskontrollen der operativen Arbeiten durchgeführt (Varcoe 2000, S. 120). 


\section{Instrumente}

Zur Bewältigung dieser Aufgaben wurden bereits im Vorfeld und auch im Nachgang zur Arbeit der Corporate Real Estate Portfolio Alliance Instrumente entwickelt. ${ }^{4}$ Im Folgenden werden nun ausgewählte Ansätze in den Prozess eingeordnet und in ihrer Funktion beschrieben.

\section{Prognose und Definition der Nutzerbedarfe}

Die Kenntnis der Nutzerbedarfe ist die Voraussetzung zur Bestimmung des Flächenbestands, der den Nutzungsanforderungen optimal entspricht. Dies gestaltet sich in der Regel bei privaten und öffentlichen Unternehmen sehr schwierig, da die Nutzer ihre Bedarfe selbst schwer einschätzen können bzw. selbst nur einen sehr kurzen Planungshorizont haben. Häufig wird diese Unsicherheit auch als Argument genutzt für eine sehr passive, fatalistische Haltung der Immobilienverwalter gegenüber drohenden Risiken (z. B. Leerstände in Objekten mit auslaufender Nutzung).

Um dieser Unsicherheit zu begegnen, wurde im Rahmen der Corporate Real Estate Portfolio Alliance ein mehrstufiges Modell zur Bedarfsprognose entwickelt. ${ }^{5}$ Die vier Stufen sind als aufeinander aufbauende Schritte zu verstehen, je nach Zunahme der Unsicherheit:

- $\quad$ Stufe 1: Direkte Übersetzung von Daten aus dem Kerngeschäft - einfache Abfragen bei Nutzern, Eignung überwiegend nur bei Unternehmen mit stabilen Umweltbedingungen oder für sehr kurze Prognosezeiträume (bis 18 Monate).

- Stufe 2: Extrapolation von Geschäftsindikatoren - für Prognosezeiträume über 18 Monaten bis 3 Jahre können Veränderungen der Flächenbedarfe auf Basis von Wachstumseinschätzungen im Kerngeschäft extrapoliert werden.

- Stufe 3: Szenariotests - Im Forschungsprojekt der TU Delft wurde eigens für das Immobilienmanagement der öffentlichen Hand ein Szenarioplanungsansatz entwickelt. Eine detaillierte Beschreibung findet sich bei Dewulf und Van der Schaaf (Dewulf/Van der Schaaf 1998, S. 19 f.; Dewulf/Van der Schaaf 2004, S. 164 f.).

- Stufe 4: „Un-forecasting“ (keine Prognosen) - basierend auf der Erkenntnis, dass Prognosen nicht in ausreichender Qualität möglich sind, sind Vorkehrungen zu treffen, das Flächenangebot möglichst flexibel zu gestalten. Beispielhaft seien hier Sonderkündigungsrechte und Verlängerungsoptionen für (Rück-)Mietverträge genannt.

4 Vgl. Definition von Controllinginstrumenten als Führungsinstrumente in Schäffer/Steiners 2003, S. 4 f.

5 Für eine detaillierte Beschreibung siehe O’Mara 2000, S. 123 f. 


\section{Analyse}

Konnten die Nutzerbedarfe prognostiziert werden, beschäftigt man sich in der nächsten Phase mit der Analyse des Bestands- und Zielportfolios (Ist- und Soll-Zustand).

Um sich von der kritisierten Bottom-Up-Herangehensweise zu lösen, ist ein abstrakterer Weg der Portfoliostrukturierung zu beschreiten. Im Gegensatz zur Beschäftigung mit Einzelobjekten und deren emotionalen Komponenten, gilt es Objekte den Objektgruppen zuzuordnen. Hierzu gibt es unterschiedliche Ansätze, die aus Sicht des Autors alle einen Mehrwert gegenüber der Beschäftigung mit Einzeladressen bieten.

Eine erste Clusterung sieht folgende Objektgruppen vor (Wills 2007, S. 2):

- Strategische Objekte: alle Immobilien, die das Unternehmen für die Erfüllung seiner langfristigen Immobilienstrategie benötigt.

- „Flagship“ Objekte: Liegenschaften, die die Kultur, das Image des Unternehmens darstellen.

- Kernobjekte: alle mittelfristig betriebsnotwendigen Objekte zur Nutzung durch Kerngeschäftseinheiten.

- Periphere Objekte: kurzfristig betriebsnotwendige Objekte aufgrund zyklischer Nutzerbedarfe.

- Entbehrliche Objekte: aktuell oder mittelfristig nicht mehr benötigte Liegenschaften.

Abschließend ist noch der Ansatz von Gibson und Louargand zu nennen, der einen analogen Strukturierungsansatz in Cluster auf Basis der Einschätzung des Einflusses der Immobilien auf das strategische Risiko verfolgt (Für eine Zusammenfassung siehe Pfirsching 2007, S. 61 f.).

\section{Strategieentwicklung}

Der Mehrwert der Portfoliostrukturierung liegt in der Möglichkeit, für größere, homogene Teilportfolien (die Cluster) mit Hilfe von vorab definierten Normstrategien ${ }^{6}$ schnell zu einer Strategieentscheidung für viele Objekte $\mathrm{zu}$ kommen (Top-Down-Herangehensweise), ohne sich in Diskussionen zu Einzelobjekten zu verlieren (Bottom-Up-Herangehensweise).

So positioniert die öffentliche Immobilienverwaltung der Niederlande ihre Kernobjekte in einer BCG-Matrix. ${ }^{7}$ Im Sinne des priorisierten Ziels Risikovermeidung entscheidet man sich bei den „Dog“-Objekten mit hohem Risiko und niedriger Rendite für den Verkauf. Präferiert wird dabei die Möglichkeit, sich bei noch mittelfristig laufender Nutzung in Form einer Sale and Rentback-Transaktion ${ }^{8}$ von diesen Objekten zu trennen, um den

6 Z. B. entbehrliche Objekte werden verkauft, bei Kernobjekten ist die Wirtschaftlichkeit von Eigentum als Bereitstellungsform zu prüfen.

7 Portfoliomatrix für das strategische Management, entwickelt von Boston Consulting Group (BCG), die anhand der Kriterien Marktwachstum und relativer Marktanteile vier Quadranten unterscheidet. „Poor dogs“ sind z. B. Produkte mit unterdurchschnittlicher Ausprägung beider Kriterien.

8 Für eine Abgrenzung von Sale and Rentback und Sale and Leaseback siehe Pfirsching 2007, S. 51 f. 
höheren Marktwert mit Rückmietvertrag zu erlösen (Van der Schaaf/De Puy 2001, S. 157).

In Ergänzung zu diesen Portfolioansätzen mit Normstrategien bietet sich die Nutzung eines Best Practice-Strategieentwicklungs- und -entscheidungsprozesses an, der für die öffentliche Immobilienverwaltung in USA vorgeschlagen wurde (siehe Abbildung 3).

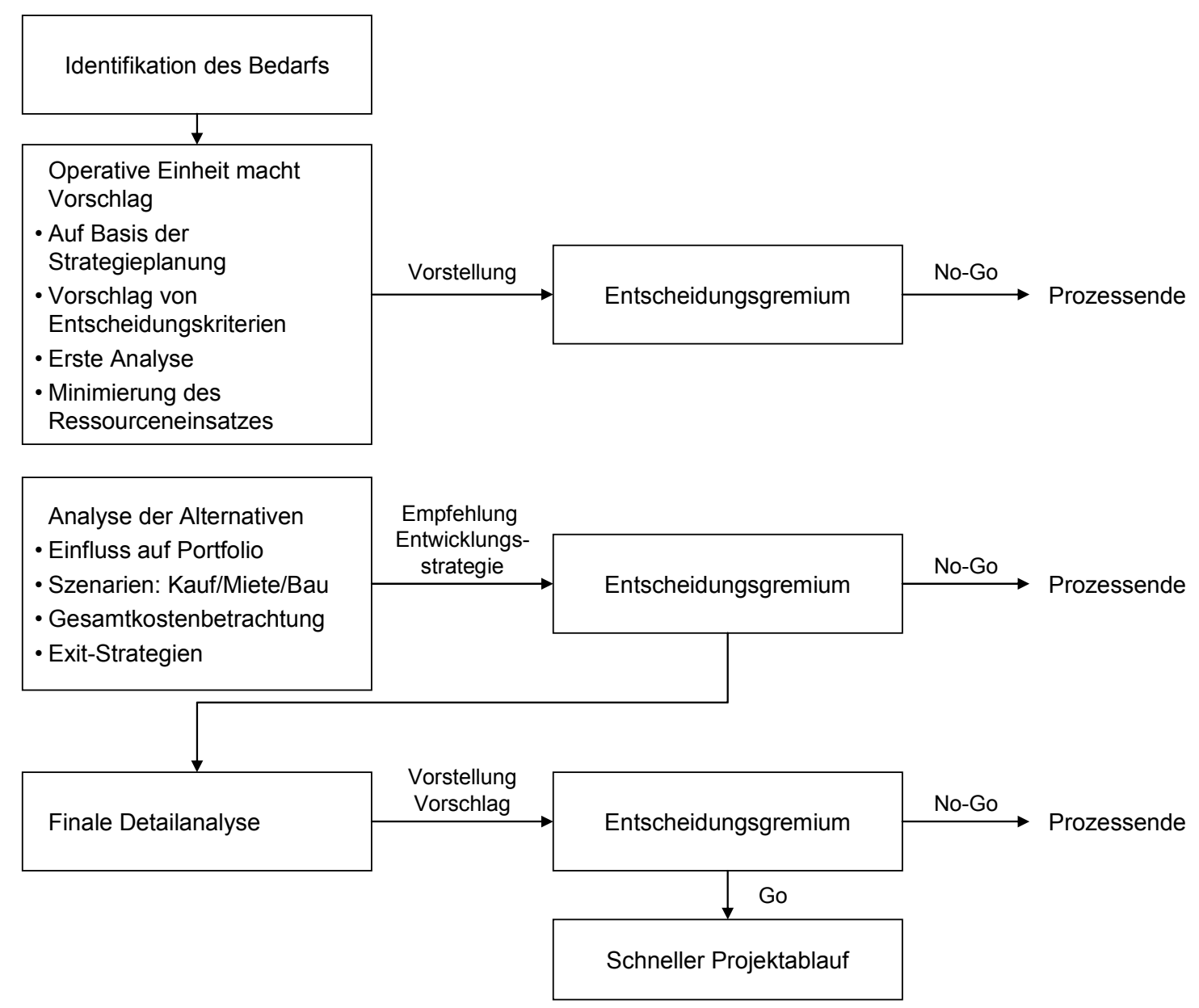

\section{Abb. 3: Typischer Entscheidungsprozess für Immobilienentscheidungen}

Quelle: $\quad$ The National Academy of Sciences 2004, S. 56

\section{Umsetzungsplanung}

Im Rahmen der Umsetzungsplanung gilt es dann, geeignete Maßnahmen zur Umsetzung der Strategien zu finden. Sind diese bei den Portfolioansätzen mit den Normstrategien bereits für spezielle Fälle definiert, bietet die im Folgenden beispielhaft vorgestellte, von Nourse und Roulac (1993) entwickelte Matrix Orientierung, die Immobilienzielen passende Maßnahmen zuordnet.

Die gesamte Matrix umfasst 14 Maßnahmen, die 8 Immobilienzielen zugeordnet werden. Im Folgenden sollen anhand eines Immobilienziels die Aussagen der Matrix vorgestellt werden. Die Minimierung der Flächenbereitstellungskosten umfasst alle immobilienbezogenen Kosten: Flächen- und Bewirtschaftungskosten. Bei der Standortwahl als erster 
Maßnahme kann die Minimierung erreicht werden, indem Objekte an Standorten gewählt werden, deren Miete bzw. Anschaffungskosten vergleichsweise gering sind. Der Flächenumfang kann minimiert werden, indem mehr Mitarbeiter im Objekt untergebracht werden und somit der Flächenverbrauch pro Mitarbeiter sinkt. Die Miet- bzw. Anschaffungs- und Bewirtschaftungskosten können sinken, indem eine einfache Ausstattung gewählt wird. Problematisch wird die Erreichung des Immobilienziels bei der Kontrolle über das Objekt (in Abgrenzung von Ansprüchen anderer Nutzer). Die Maximierung des Kontrollumfangs geht in der Regel mit einer Steigerung der Flächenbereitstellungskosten einher.

\begin{tabular}{|c|c|c|c|c|}
\hline \multirow[b]{2}{*}{$\begin{array}{l}\text { Operative } \\
\text { Maßnahmen }\end{array}$} & \multicolumn{4}{|c|}{ Strategische Immobilienziele } \\
\hline & $\begin{array}{l}\text { Minimierung } \\
\text { Flächenbereit- } \\
\text { stellungskosten }\end{array}$ & $\begin{array}{l}\text { Erhöhung } \\
\text { der } \\
\text { Flexibilität }\end{array}$ & $\begin{array}{c}\text { Erhöhung } \\
\text { individuelle } \\
\text { Nutzer- } \\
\text { zufriedenheit }\end{array}$ & $\begin{array}{l}\text { Werbewirksamkeit } \\
\text { (für operatives } \\
\text { Geschäft) }\end{array}$ \\
\hline Standortwahl & $\begin{array}{l}\text { Dezentrale, weniger } \\
\text { populäre Standorte }\end{array}$ & $\begin{array}{l}\text { Weniger } \\
\text { populäre } \\
\text { Standorte }\end{array}$ & $\begin{array}{c}\text { Nähe zu } \\
\text { Wohnorten der } \\
\text { Mitarbeiter }\end{array}$ & $\begin{array}{c}\text { Prestige-Objekte, } \\
\text { sehr gute } \\
\text { Visibilität } \\
\end{array}$ \\
\hline Flächenumfang & $\begin{array}{l}\text { Minimierung des } \\
\text { Flächenverbrauchs } \\
\text { pro Mitarbeiter }\end{array}$ & & $\begin{array}{l}\text { Erhöhung des } \\
\text { Flächen- } \\
\text { verbrauchs pro } \\
\text { Mitarbeiter }\end{array}$ & \\
\hline Flächenausstattung & $\begin{array}{c}\text { Einfache } \\
\text { Ausstattung }\end{array}$ & & $\begin{array}{c}\text { Gute } \\
\text { Ausstattung } \\
\end{array}$ & \\
\hline Gebäudetechnik & & & $\begin{array}{l}\text { Wert auf } \\
\text { Komfort } \\
\text { legen }\end{array}$ & \\
\hline Kontrollumfang & $\begin{array}{l}\text { Keine gleichzeitige } \\
\text { Zielerreichung mög- } \\
\text { lich }\end{array}$ & & & \\
\hline
\end{tabular}

Abb. 4: Auszug aus der Zuordnungsmatrix der operativen Maßnahmen zu strategischen Immobilienzielen

Quelle: $\quad$ Auszug aus Nourse/Roulac 1993, S. 490 f.

\section{Monitoring und Verbesserung}

Parallel zu den vier Hauptphasen des Portfoliomanagementprozesses verläuft der kontinuierliche Monitoring- und Verbesserungsprozess. Hierzu hat Seilheimer (2007) in einer aktuellen Dissertation ein Portfoliomanagementsystem auf Basis von Benchmarks speziell für die öffentliche Hand entwickelt. In Anlehnung an die Balanced Scorecard schlägt er Muster-Portfolio Scorecards für mehrere Perspektiven vor (siehe Abbildung 5 für die Finanzperspektive), mit deren Hilfe der Erfolg der Maßnahmenumsetzung kontinuierlich überprüft und an externen Benchmarks gemessen werden kann.

Abschließend ist zu bemerken, dass die Strategieentwicklung und Maßnahmendefinition keine einmalige Aufgabe ist, sondern sich an die ändernden Umweltbedingungen anpas- 
sen muss. Gerade weil Investitionen in Immobilien einen langfristigen Charakter haben, muss das Portfoliomanagement für selbstgenutzte Immobilien frühzeitig Veränderungen antizipieren und entsprechende Maßnahmen anstoßen. Sonst drohen nicht nutzungsadäquate Flächen für die Nutzer, daraus resultierender Leerstand und Wertverlust, gerade bei nicht marktgängigen Liegenschaften ohne Folgenutzungsperspektive.

\begin{tabular}{|l|l|l|l|l|}
\hline Nr. & Strategisches Ziel & Messgröße & Zielwert & $\begin{array}{l}\text { Strategische } \\
\text { Maßnahme }\end{array}$ \\
\hline F1 & $\begin{array}{l}\text { Abbau des } \\
\text { Instandhaltungsstaus }\end{array}$ & $\begin{array}{l}\text { Rel. } \\
\text { Instandhaltungsstau }\end{array}$ & $\begin{array}{l}\text { Int./ ext. } \\
\text { Benchmarks }\end{array}$ & $\begin{array}{l}\text { Aufgabe maroder } \\
\text { Liegenschaften, Abbau } \\
\text { durch Maßnahmen- } \\
\text { bündelung }\end{array}$ \\
\hline F2 & $\begin{array}{l}\text { Erzielung } \\
\text { marktüblicher Bereit- } \\
\text { stellungskosten }\end{array}$ & $\begin{array}{l}\text { Rel. } \\
\text { Bereitstellungskosten }\end{array}$ & $\begin{array}{l}\text { Int./ ext. } \\
\text { Benchmarks }\end{array}$ & $\begin{array}{l}\text { Energieoptimierung } \\
\text { durch aktives Energie- } \\
\text { management }\end{array}$ \\
\hline F3 & $\begin{array}{l}\text { Kostendeckende } \\
\text { Verrechnungsmiete }\end{array}$ & $\begin{array}{l}\text { Rel. Mietzins je } \\
\text { Flächenart }\end{array}$ & $\begin{array}{l}\text { Int./ ext. } \\
\text { Benchmarks }\end{array}$ & $\begin{array}{l}\text { Durchsetzung von } \\
\text { kostendeckenden } \\
\text { Verrechnungsmieten, } \\
\text { ggf. mit Bonus-Malus- } \\
\text { Regelung }\end{array}$ \\
\hline
\end{tabular}

Abb. 5: Muster-Portfolio Scorecard-Finanzperspektive

Quelle: $\quad$ Seilheimer 2007, S. 107

\section{Ausblick}

Die Einführung eines effektiven Portfoliomanagements ist nur ein erster Schritt zur Optimierung des Immoblienmanagements für selbstgenutzte Immobilien. Auf Ebene des Asset Managements sind aus der praktischen Erfahrung des Autors zumindest noch zwei Voraussetzungen zu schaffen.

Um immanenten Interessenskonflikten zwischen dem nach Flächenreduzierung strebenden Immobilieneigentümer, dem nach Maximierung der Flächen und somit Aufgaben strebenden Immobilienverwalter sowie den Nutzern innerhalb der Organisation des Selbstnutzers zu begegnen, ist eine Funktionentrennung zwischen Portfolio- und Asset Management auf der einen Seite (Ebene 2 und 3 der Pyramide) und der operativen Verwaltung (Ebene 4) zu realisieren. Die konfligierenden Interessen werden dann durch ein Besteller-Ersteller-Verhältnis auf Basis marktüblicher Konditionen reguliert (Bongartz 2002, S. 49).

Weiterhin sollte die Harmonisierung von Eigentümer- und Nutzerinteressen durch die Einführung eines Mieter-Vermieter-Modells erfolgen. Auf Basis marktüblicher Konditionen wird so das Zusammenspiel von Flächenbereitsteller und -nutzer reguliert, eine dezentrale Ressourcenverantwortung realisiert und Anreize zur Optimierung geschaffen.

Vollständige Transparenz wird abschließend wie im Falle der US-General Services Administration (Public Buildings Fund) oder der Deutsche Telekom AG (GMG - General- 
mietgesellschaft) erreicht, wenn die Immobilien auf ein Sondervermögen übertragen werden, das nur Einnahmen aus der Vermietung und Bewirtschaftung erzielt und die gesamten Bereitstellungskosten aus diesen Einnahmen tragen muss. ${ }^{9}$

Im Zusammenspiel von effektivem Portfoliomanagement im Sinne der Eigentümer- und Nutzerinteressen, Funktionentrennung und Mieter-Vermieter-Modell wird so ein sich selbst regulierendes und nach ständiger Optimierung strebendes Immobilienmanagementsystem für selbstgenutzte Immobilien installiert.

\section{Literaturverzeichnis}

Belau, Michael, Michael Schildger und Verena Sturm (2007), Immobiliencontrolling - Optimierung der immobilienbezogenen Kosten durch Gründung einer Generalmietgesellschaft, in: Controlling \& Management, 51. Jg., Heft 2, S. 110-116.

Bomba, Tom (2000), The Alliance Program: Establishing a new knowledge base on the corporate real estate portfolio management competency, in: Journal of Corporate Real Estate, vol. 2., no. 2, pp. 103-112.

Bongartz, Oliver (2002), Immobilienmanagement statt Verwaltung der Liegenschaften - Freie und Hansestadt Bremen optimiert das Grundvermögen, in: Innovative Verwaltung, 24. Jg., Heft 1/2, S. 46-49.

Dewulf, Geert and Pity van der Schaaf (1998), Portfolio management in the midst of uncertainties: How scenario planning can be useful, in: Journal of Corporate Real Estate, vol. 1, no. 1, pp.19-28.

Dewulf, Geert and Pity van der Schaaf (2004), Scenario Planning to Define a Public Real Estate Strategy, in: Nordic Journal of Surveying and Real Estate Research, Vol 1, no. 2, pp. 164-174.

Duckworth, Steven L. (1993), Realizing the Strategic Dimension of Corporate Real Property Through Improved Planning and Control Systems, in: Journal of Real Estate Research, vol. 8, no. 4, pp. 495-509.

Ecke, Christian (2004), Strategisches Immobilienmanagement der öffentlichen Hand - empirische Untersuchungen und Handlungsempfehlungen, Köln.

Evers, Frans, Pity van der Schaaf and Geert Dewulf (2002), Public Real Estate: Succesful Management Strategies, Delft.

Gibson, Virginia A. and Marc Louargand (2002), Risk Management and the Corporate Real Estate Portfolio, Konferenzbeitrag American Real Estate Society Annual Meeting 2002.

Nourse, Hugh O. and Stephen E. Roulac (1993), Linking Real Estate Decisions to Corporate Strategy, in: Journal of Real Estate Research, vol. 8, no. 4, pp. 475-494.

O’Mara, Martha A. (2000), Strategies for demand forecasting in corporate real estate portfolio management, in: Journal of Corporate Real Estate, vol. 2, no. 2, pp. 123-137.

Pfirsching, Frank (2007), Portfoliotransaktionen von Selbstnutzern - eine immobilienwirtschaftliche Analyse, Wiesbaden.

Roulac, Stephen E. (2001), Corporate Property Strategy is Integral to Corporate Business Strategy, in: Journal of Real Estate Research, vol. 22, no. 1/2, pp. 129-152.

Schäffer, Utz (2004), Zum Verhältnis von Unternehmensethik und Controlling, in: European Business School Working Papers on Management Accounting \& Control, no. 12.

Schulte, Karl-Werner, Wolfgang Schäfers, Eleonore Pöll und Markus Amon (2006), Grundlagen des Immobilienmanagements der öffentlichen Hand, in: Schulte, Karl-Werner und Wolfgang Schäfers und Eleonore Pöll und Markus Amon, Handbuch Immobilienmanagement der öffentlichen Hand, Köln, S. 21-36.

Seilheimer, Stephan (2007), Immobilien-Portfoliomanagement für die öffentliche Hand - Ziele, Nutzen und Vorgehen in der Praxis auf der Basis von Benchmarks, Wiesbaden.

Szigeti, Francoise and Gerald Davis (2000), The Value Chain for Corporate Real Estate, Arbeitspapier International Centre for Facilities.

The National Academy of Sciences (2004), Investments in Federal Facilities: Asset Management Strategies for the $21^{\text {st }}$ Century, Washington.

Van der Schaaf, Pity and Lydia de Puy (2001), CRE portfolio management: Improving the process, in: Journal of Corporate Real Estate, vol. 3, no. 2, pp. 150-160.

Varcoe, Barry (2000), A process for the portfolio management of real estate assets, in: Journal of Corporate Real Estate, Vol 2, No 2, pp. 113-122.

Wills, Peter (2007), Transaction Based Corporate Real Estate Management, Konferenzbeitrag 13. Pacific-Rim Real Estate Society Conference, Freemantle.

9 Bongartz 2002, S. 47; Belau/Schildger/Sturm 2007, S. 114; Van der Schaaf/De Puy 2001, S. 153. 